\title{
BOUNDARY VALUE PROBLEMS FOR EQUATIONS OF VISCOUS HEAT-CONDUCTING GAS IN TIME-INCREASING NON-CYLINDRICAL DOMAINS
}

\author{
I.A. KALIEV, A.A. SHUKHARDIN, G.S. SABITOVA
}

\begin{abstract}
In this paper we prove global solvability of the initial-boundary value problems for the complete system of equations describing one-dimensional non-stationary flow of the viscous heat-conducting gas in time-increasing non-cylindrical domains. Local existence and uniqueness of these problems are proved in earlier articles by Kazhikhov A.V. and Kaliev I.A. This is why, the proof of the global in time existence and uniqueness theorem is connected with obtaining a priori estimates, in which the constant depend only on the data of the problem and the value of the time interval $T$, but do not depend on the period of existence of a local solution. The study is made in terms of Eulerian variables.
\end{abstract}

Keywords: Navier-Stokes equations system, heat-conducting gas, global solvability, timeincreasing non-cylindrical domains.

Mathematics Subject Classification: 35Q30, 76D05,76N10

\section{INTRODUCTION}

The complete system of equations of viscous heat-conduction gas motion, or Navier-Stokes system of equations is an interesting and important class of partial differential equations. In the theory of such systems, one of the central problems is the unique solvability "in general" both in time and the data.

The study of issues on well-definiteness of initial-boundary value problems for Navier-Stokes system of equations began with the work by J. Serrin in 1959 [1]. There were formulation the main boundary value problems and proven uniqueness theorems in the class of smooth functions. We also mention an earlier paper by D. Graffi in 1953 [2] on the uniqueness of classical solutions for a barotropic gas.

The first result on solvability of Navier-Stokes equations was obtained by J. Nash in 1962 [3]. He showed the existence of classical solution to Cauchy problem for small times. By other methods, this result was reproduced and generalized in works by N. Itaya [4, A.I. Volpert and S.I. Khudyaev [5].

For initial boundary value problems theorem on local in time existence and uniqueness were proven by V.A. Solonnikov [6] and A. Tani [7].

The first result on the unique global solvability in time and in data was established by Ya.I. Kanel in 1968 [8] for the Cauchy problem for the equations of one-dimensional viscous barotropic gas $\left(p=R \rho^{\gamma}\right)$. For the Bürgers model ( $p=$ const) the solvability of Cauchy problem and initial boundary value problems was proven in works by N. Itaya [9, [10] and A. Tani [11.

In 1976, A.V. Kazhikhov [12] first obtained the result on global solvability for the equations of one-dimensional viscous heat-conducting gas. In what follows in a series of works by A.V. Kazhikhov [13]-[16], V.V. Shelukhin [17]-[19], S.Ya. Belov [20], V.A. Vaigant [21], 22] there was constructed a rather complete theory on global solvability of main initial boundary value problems and Cauchy problem for equations of viscous gas motion.

I.A. Kaliev, A.A. Shukhardin, G.S. Sabitova, Boundary value problems for equations of VISCOUS HEAT-CONDUCTING GAS IN TIME-INCREASING NON-CYLINDRICAL DOMAINS.

(c) Kaliev I.A., Shukhardin A.A., Sabitova G.S. 2014.

Submitted July 4, 2014 . 
In works by I.A. Kaliev and A.V. Kazhikhov [23], 24] there were studied issues on the unique solvability of a problem with a free boundary modeling the process of phase transition between a viscous gas and a solid state. At that, there appears an auxiliary problem describing the motion of a viscous heat-conducting gas in a curvilinear domain and the existence and the unique solvability of local solution was proven.

As a rule, the domain in which one proves the global existence in time, is either the strip $\{(x, t) \mid-$ $\infty<x<\infty, 0<t<T\}$ or a cylinder $\{(x, t) \mid a<x<b, 0<t<T\} ; a, b, T$ are given constants. In our work, for the complete system of equations of one-dimensional non-stationary motion of a viscous heat-conducting gas, we prove the global solvability of initial boundary value problems in non-cylindrical domains $\{(x, t) \mid 0<x<s(t), 0<t<T\}$ increasing with time; here $x=s(t)$ is a given smooth increasing function.

For a viscous gas, the results on global solvability are known for the problem with a free boundary on gas outflow into vacuum [12, [25] and for the problem on a piston moving by a prescribed law [25]. In both the problems the velocity of boundary $s(t)$ of domain occupied by the gas coincided with the velocity of the with the coordinates $s(t)$, i.e., $u(s(t), t)=d s(t) / d t, 0<t<T$. In other words, the gas did not flow through the boundary $s(t)$ and this fact played an essential role in the proof of the existence theorems since the domain of the solution becomes a fixed cylinder in Lagrangian coordinates.

In the present work $u(s(t), t)=0, d s(t) / d t>0$, i.e., $u(s(t), t)-d s(t) / d t<0$, and the gas inflows via the moving boundary of the domain $x=s(t)$. We make the study in terms of Eulerian variables.

The case $d s(t) / d t \leqslant 0$ was considered in works by I.A. Kaliev and M.S. Pokuiko [26], [27].

\section{Formulation of the PROBlem AND the MAin RESUlts}

Given a smooth function $x=s(t)$, we suppose that a non-cylindrical domain $\Omega_{T}=\{(x, t) \mid 0<x<$ $s(t), 0<t<T\}$, is occupied by a viscous heat-conducting gas. In the work we study the case when the domain widens in time, i.e., $d s(t) / d t>0$. The one-dimensional non-stationary motion of a viscous heat-conducting gas in domain $\Omega_{T}$ is described by the system of equations [25]

$$
\begin{aligned}
& \frac{\partial \rho}{\partial t}+\frac{\partial(\rho u)}{\partial x}=0, \quad(x, t) \in \Omega_{T}, \\
& \rho\left(\frac{\partial u}{\partial t}+u \frac{\partial u}{\partial x}\right)=\mu \frac{\partial^{2} u}{\partial x^{2}}-\frac{\partial p}{\partial x}, \quad p=R \rho \theta, \quad(x, t) \in \Omega_{T}, \\
& \rho\left(\frac{\partial \theta}{\partial t}+u \frac{\partial \theta}{\partial x}\right)=\kappa \frac{\partial^{2} \theta}{\partial x^{2}}+\mu\left(\frac{\partial u}{\partial x}\right)^{2}-p \frac{\partial u}{\partial x}, \quad(x, t) \in \Omega_{T} .
\end{aligned}
$$

Here $\rho(x, t), u(x, t), p(x, t)$ and $\theta(x, t)$ are the density, velocity, pressure and absolute temperature of the gas; $\mu, R, \kappa$ are positive constants denoting the viscosity, gas constant, and heat conductivity factor, respectively.

At the initial time we specify $u, \theta, \rho$ :

$$
u(x, t)]\left.\right|_{t=0}=u_{0}(x),\left.\quad \theta(x, t)\right|_{t=0}=\theta_{0}(x),\left.\quad \rho(x, t)\right|_{t=0}=\rho_{0}(x), \quad x \in\left[0, s_{0}\right],
$$

where $s_{0}=s(0)$. On the known boundaries $x=0$ and $x=s(t)$ we impose the conditions

$$
\begin{aligned}
& \left.u(x, t)\right|_{x=0}=0,\left.\quad u(x, t)\right|_{x=s(t)}=0, \quad t \in[0, T], \\
& \left.\theta(x, t)\right|_{x=0}=\theta_{1}(t),\left.\quad \theta(x, t)\right|_{x=s(t)}=\theta_{2}(t), \quad t \in[0, T], \\
& \left.\rho(x, t)\right|_{x=s(t)}=\rho_{2}(t), \quad t \in[0, T] .
\end{aligned}
$$

We assume that the inequalities

$$
\begin{aligned}
& 0<m \leqslant \rho_{0}(x), \rho_{2}(t), \theta_{0}(x), \theta_{1}(t), \theta_{2}(t) \leqslant M<+\infty, \\
& 0<s_{0}, \quad 0<m \leqslant \frac{d s}{d t}(t) \leqslant M,
\end{aligned}
$$

hold true for each $t \in[0, T]$ and $x \in\left[0, s_{0}\right]$, where $m, M$ are some positive constants.

Problem Gas. Find functions $\rho(x, t), u(x, t), \theta(x, t)$ solving system of equations (1)-(3), if the initial conditions and boundary conditions (4)-(7) are satisfied. 
Theorem 1. Suppose that initial and boundary data in problem Gas belong to Hölder spaces

$$
\begin{array}{lll}
\rho_{0}(x) \in C^{1+\alpha}\left(\left[0, s_{0}\right]\right), & u_{0}(x) \in C^{2+\alpha}\left(\left[0, s_{0}\right]\right), & \theta_{0}(x) \in C^{2+\alpha}\left(\left[0, s_{0}\right]\right), \\
s(t), \rho_{2}(t) \in C^{1+\alpha}([0, T]), & \theta_{1}(t), \theta_{2}(t) \in C^{(2+\alpha) / 2}([0, T]), &
\end{array}
$$

$0<\alpha=$ const $<1$; conditions (8), (9) and conditions of zero order and first order matching at the points $(0,0),\left(s_{0}, 0\right)$ are satisfied. Then problem Gas has the unique classical solution possessing the properties

$$
\rho(x, t) \in C^{1+\alpha}\left(\bar{\Omega}_{T}\right), \quad u(x, t) \in C^{2+\alpha,(2+\alpha) / 2}\left(\bar{\Omega}_{T}\right), \quad \theta(x, t) \in C^{2+\alpha,(2+\alpha) / 2}\left(\bar{\Omega}_{T}\right),
$$

at that,

$$
0<m_{1} \leqslant \rho(x, t) \leqslant M_{1}<+\infty, \quad 0<m_{2} \leqslant \theta(x, t) \leqslant M_{2}<+\infty, \quad(x, t) \in \Omega_{T} ;
$$

where $m_{1}, M_{1}, m_{2}, M_{2}$ are some positive constants.

Local existence and uniqueness theorem for problem Gas was proven in [23], 24]. This is why the proof of the above theorem is related with obtaining apriori estimates, where constants depend only on data of the problem and time interval $T$ but are independent of the existence interval for the local solution.

\section{AUXILIARY PROPOSITIONS AND APRIORI ESTIMATES}

We assume that $\rho(x, t)>0, \theta(x, t)>0$ (there is a local in time existence theorem with appropriate estimates) [23], [24].

Lemma 1. For each $t \in[0, T]$, the estimates

$$
\int_{0}^{s(t)} \rho(x, t) d x=\int_{0}^{s_{0}} \rho_{0}(x) d x+\int_{0}^{t} \rho_{2}(\tau) \frac{d s(\tau)}{d \tau} \leqslant M_{0}
$$

where

$$
M_{0}=\int_{0}^{s_{0}} \rho_{0}(x) d x+\int_{0}^{T} \rho_{2}(\tau) \frac{d s(\tau)}{d \tau} d \tau .
$$

Proof. Employing conditions (5), (7), integrate equation (1) w.r.t. $x$ from 0 to $s(t)$ :

$$
\frac{d}{d t} \int_{0}^{s(t)} \rho(x, t) d x-\rho_{2}(t) \frac{d s}{d t}=0 .
$$

Integrating w.r.t. $t$, we complete the proof:

$$
\begin{aligned}
& \int_{0}^{s(t)} \rho(x, t) d x=\int_{0}^{s_{0}} \rho_{0}(x) d x+\int_{0}^{t} \rho_{2}(\tau) \frac{d s(\tau)}{d \tau} d \tau \leqslant M_{0}, \\
& M_{0}=\int_{0}^{s_{0}} \rho_{0}(x) d x+\int_{0}^{T} \rho_{2}(\tau) \frac{d s(\tau)}{d \tau} d \tau .
\end{aligned}
$$

In what follows, while obtaining estimates for functions $\rho, u, \theta$ in a domain occupied by a viscous gas, we employ the methods developed by B.A. Vaigant [21. We observe that in 21 the domain occupied by gas is the rectangle $(0,1) \times(0, T)$, while in our case the domain occupied by gas is the curvilinear trapezoid $\Omega_{T}=\{(x, t) \mid 0<x<s(t), 0<t<T\}$, where $x=s(t)$ is a given increasing function. Nevertheless, we succeed to prove all desired apriori estimates.

In $\Omega_{T}$ we introduce an auxiliary function $B(x, t)$ as follows:

$$
\begin{aligned}
& \frac{\partial B}{\partial x}=\frac{1}{\mu} \rho u, \quad \frac{\partial B}{\partial t}=\frac{\partial u}{\partial x}-\frac{1}{\mu} R \rho \theta-\frac{1}{\mu} \rho u^{2}, \\
& \left.B\right|_{t=0}=B_{0}(x)=\frac{1}{\mu} \int_{0}^{x} \rho_{0}(\xi) u_{0}(\xi) d \xi, \quad 0 \leqslant x \leqslant s_{0} .
\end{aligned}
$$


In [21] for function $B(x, t)$ there were obtained the identities

$$
\begin{aligned}
& \frac{\partial}{\partial t}(B+\ln \rho)+u \frac{\partial}{\partial x}(B+\ln \rho)+\frac{1}{\mu} R \rho \theta=0, \\
& \frac{\partial}{\partial t}\left(\rho e^{B}\right)+u \frac{\partial}{\partial x}\left(\rho e^{B}\right)+\frac{1}{\mu} R \rho^{2} \theta e^{B}=0, \\
& \frac{\partial}{\partial t}\left(\frac{1}{\rho} e^{-B}\right)+u \frac{\partial}{\partial x}\left(\frac{1}{\rho} e^{-B}\right)-\frac{1}{\mu} R \theta e^{-B}=0 .
\end{aligned}
$$

Lemma 2. There exists a constant $C$ depending on boundary data and $T$ such that for each $(x, t) \in$ $\Omega_{T}$ the inequality

$$
|B(x, t)| \leqslant C\left(1+\left(\int_{0}^{s(t)} \rho u^{2} d x\right)^{1 / 2}+\int_{0}^{t} \int_{0}^{s(\tau)} \rho \theta d x d \tau+\int_{0}^{t} \int_{0}^{s(\tau)} \rho u^{2} d x d \tau\right)
$$

holds true.

Proof. We integrate function $\partial B / \partial t$ over the domain $\Omega_{t}=\{(x, \tau) \mid 0<x<s(\tau), 0<\tau<t\}$. Sometimes it will be more convenient for us to write domain $\Omega_{t}$ as $\Omega_{t}=\{(x, \tau) \mid 0<x<s(t), h(x)<\tau<t\}$, where $h(x)=0$ as $x \in\left[0, s_{0}\right]$ and $s(h(x))=x$ as $s_{0}<x \leqslant s(t)$. We have

$$
\begin{aligned}
\iint_{\Omega_{t}} B_{t} d \tau d x & =\left.\int_{0}^{s_{0}} B(x, \tau)\right|_{\tau=0} ^{\tau=t} d x+\left.\int_{s_{0}}^{s(t)} B(x, \tau)\right|_{\tau=h(x)} ^{\tau=t} d x \\
& =\int_{0}^{s_{0}} B(x, t) d x-\int_{0}^{s_{0}} B(x, 0) d x+\int_{s_{0}}^{s(t)} B(x, t) d x-\int_{s_{0}}^{s(t)} B(x, h(x)) d x \\
& =\int_{0}^{s(t)} B(x, t) d x-\frac{1}{\mu} \int_{0}^{s_{0}} \int_{0}^{x} \rho_{0}(\xi) u_{0}(\xi) d \xi d x-\int_{s_{0}}^{s(t)} B(x, h(x)) d x .
\end{aligned}
$$

On the other hand,

$$
\begin{aligned}
\iint_{\Omega_{t}} B_{t} d x d \tau & =\iint_{\Omega_{t}}\left(u_{x}-\frac{1}{\mu} R \rho \theta-\frac{1}{\mu} \rho u^{2}\right) d x d \tau \\
& =-\frac{R}{\mu} \int_{0}^{t} \int_{0}^{s(\tau)} \rho \theta d x d \tau-\frac{1}{\mu} \int_{0}^{t} \int_{0}^{s(\tau)} \rho u^{2} d x d \tau .
\end{aligned}
$$

As a result we get

$$
\begin{aligned}
\int_{0}^{s(t)} B(x, t) d x= & \frac{1}{\mu} \int_{0}^{s_{0}} \int_{0}^{x} \rho_{0}(\xi) u_{0}(\xi) d \xi d x+\int_{s_{0}}^{s(t)} B(x, h(x)) d x \\
& -\frac{R}{\mu} \int_{0}^{t} \int_{0}^{s(\tau)} \rho \theta d x d \tau-\frac{1}{\mu} \int_{0}^{t} \int_{0}^{s(\tau)} \rho u^{2} d x d \tau .
\end{aligned}
$$

By (8), (9) it implies the inequality

$$
\begin{aligned}
\left|\int_{0}^{s(t)} B(x, t) d x\right| \leqslant & \frac{s_{0}^{2} M}{2 \mu} \max _{x \in\left[0, s_{0}\right]}\left|u_{0}(\xi)\right|+\frac{R}{\mu} \int_{0}^{t} \int_{0}^{s(\tau)} \rho \theta d x d \tau \\
& +\frac{1}{\mu} \int_{0}^{t} \int_{0}^{s(\tau)} \rho u^{2} d x d \tau+\left|\int_{s_{0}}^{s(t)} B(x, h(x)) d x\right| .
\end{aligned}
$$

In the latter integral we change the variable $x=s(\tau), d x=\frac{d s}{d \tau} d \tau, B(x, h(x))=B(s(\tau), \tau)$ and we employ (9):

$$
\left|\int_{0}^{s(t)} B(x, t) d x\right| \leqslant C+\frac{R}{\mu} \int_{0}^{t} \int_{0}^{s(\tau)} \rho \theta d x d \tau+\frac{1}{\mu} \int_{0}^{t} \int_{0}^{s(\tau)} \rho u^{2} d x d \tau+M \int_{0}^{t}|B(s(\tau), \tau)| d \tau .
$$

Hereinafter by $C$ we denote constants depending on boundary data and $T$. 
Since for each $t \in[0, T]$ there exists a point $x_{0}=x_{0}(t) \in[0, s(t)]$ such that

$$
B\left(x_{0}(t), t\right)=\frac{1}{s(t)} \int_{0}^{s(t)} B(x, t) d x
$$

we obtain

$$
\begin{aligned}
|B(x, t)| & \leqslant\left|B\left(x_{0}(t), t\right)\right|+\int_{0}^{s(t)}\left|B_{x}\right| d x \\
& \leqslant \frac{1}{s(t)}\left|\int_{0}^{s(t)} B(x, t) d x\right|+\frac{1}{\mu}\left(\int_{0}^{s(t)} \rho d x\right)^{1 / 2}\left(\int_{0}^{s(t)} \rho u^{2} d x\right)^{1 / 2} \\
& \leqslant \frac{1}{s_{0}}\left|\int_{0}^{s(t)} B(x, t) d x\right|+\frac{1}{\mu} \sqrt{M_{0}}\left(\int_{0}^{s(t)} \rho u^{2} d x\right)^{1 / 2},
\end{aligned}
$$

$M_{0}$ is a constant in Lemma 1.

Employing Cauchy inequality, for $x=s(\tau)$ and $t=\tau$ by (15) we have

$$
|B(s(\tau), \tau)| \leqslant \frac{1}{s_{0}}\left|\int_{0}^{s(\tau)} B(x, \tau) d x\right|+\frac{M_{0}}{4 \mu^{2}}+\int_{0}^{s(\tau)} \rho u^{2} d x .
$$

We substitute (16) into (14):

$$
\left|\int_{0}^{s(t)} B(x, x)\right| \leqslant C+\frac{R}{\mu} \int_{0}^{t} \int_{0}^{s(\tau)} \rho \theta d x d \tau+\left(\frac{1}{\mu}+M\right) \int_{0}^{t} \int_{0}^{s(\tau)} \rho u^{2} d x d \tau+\frac{M}{s_{0}} \int_{0}^{t}\left|\int_{0}^{s(\tau)} B(x, \tau) d x\right| d \tau .
$$

By Grönwall inequality for the function

$$
y(t)=\left|\int_{0}^{s(t)} B(x, t) d x\right|
$$

it implies the estimate

$$
\left|\int_{0}^{s(t)} B(x, t) d x\right| \leqslant C\left(1+\int_{0}^{t} \int_{0}^{s(\tau)} \rho u^{2} d x d \tau+\int_{0}^{t} \int_{0}^{s(\tau)} \rho \theta d x d \tau\right) .
$$

Substituting this inequality in $(15)$, we complete the proof:

$$
|B(x, t)| \leqslant C\left[1+\int_{0}^{t} \int_{0}^{s(\tau)} \rho u^{2} d x d \tau+\int_{0}^{t} \int_{0}^{s(\tau)} \rho \theta d x d \tau+\left(\int_{0}^{s(t)} \rho u^{2} d x\right)^{1 / 2}\right] .
$$

Lemma 3. There exists a constant $C$ depending on boundary data and $T$ such that for each $t \in$ $[0, T]$ the inequality

$$
\int_{0}^{s(t)}(\rho \ln \rho-\rho+1) d x+\frac{R}{\mu} \int_{0}^{t} \int_{0}^{s(\tau)} \rho^{2} \theta d x d \tau \leqslant C\left(1+\max _{(x, \tau) \in \Omega_{t}}|B(x, \tau)|\right) .
$$

holds true.

Proof. We multiply equation (11) by $\rho(x, t)$ and integrate w.r.t. $x$ from 0 to $s(t)$. Then by equation (1) we get

$$
\begin{aligned}
& \frac{d}{d t} \int_{0}^{s(t)} \rho(B+\ln \rho) d x-\frac{d s(t)}{d t} \rho_{2}(t)\left[B(s(t), t)+\ln \rho_{2}(t)\right] \\
& \quad+\left.[\rho u(B+\ln \rho)]\right|_{x=0} ^{x=s(t)}+\frac{R}{\mu} \int_{0}^{s(t)} \rho^{2} \theta d x-\frac{d}{d t} \int_{0}^{s(t)} \rho d x+\frac{d s(t)}{d t} \rho_{2}(t) \pm \frac{d}{d t} \int_{0}^{s(t)} d x=0 .
\end{aligned}
$$


By (5) and (7) it yields

$$
\begin{aligned}
\frac{d}{d t} \int_{0}^{s(t)} & (\rho B+\rho \ln \rho-\rho+1) d x+\frac{R}{\mu} \int_{0}^{s(t)} \rho^{2} \theta d x \\
- & \frac{d s(t)}{d t}\left\{\rho_{2}(t)[B(s(t), t)+\ln \rho(s(t), t)-1]+1\right\}=0 .
\end{aligned}
$$

Integrating w.r.t. the time, we obtain

$$
\begin{aligned}
\int_{0}^{s(t)} & (\rho \ln \rho-\rho+1) d x+\frac{R}{\mu} \int_{0}^{t} \int_{0}^{s(\tau)} \rho^{2} \theta d x d \tau \\
= & \int_{0}^{t} \frac{d s(\tau)}{d \tau}\left[\rho_{2}(\tau) \ln \rho_{2}(\tau)-\rho_{2}(\tau)+1\right] d \tau-\int_{0}^{s(t)} \rho B d x \\
& \quad+\int_{0}^{s_{0}}\left(\rho_{0} B_{0}+\rho_{0} \ln \rho_{0}-\rho_{0}+1\right) d x+\int_{0}^{t} \frac{d s(\tau)}{d \tau} \rho_{2}(\tau) B(s(\tau), \tau) d \tau .
\end{aligned}
$$

We denote

$$
C=\int_{0}^{T} \frac{d s(\tau)}{d \tau}\left[\rho_{2}(\tau) \ln \rho_{2}(\tau)-\rho_{2}(\tau)+1\right] d \tau+\int_{0}^{s_{0}}\left(\rho_{0} B_{0}+\rho_{0} \ln \rho_{0}-\rho_{0}+1\right) d x .
$$

Taking into consideration that $\rho \ln \rho-\rho+1 \geqslant 0, d s / d t>0$, we get

$$
\begin{aligned}
\int_{0}^{s(t)}(\rho \ln \rho-\rho+1) d x+\frac{R}{\mu} \int_{0}^{t} \int_{0}^{s(\tau)} \rho^{2} \theta d x d \tau & \leqslant C+\int_{0}^{s(t)} \rho|B| d x+\int_{0}^{t} \frac{d s(\tau)}{d \tau} \rho_{2}(\tau)|B(s(\tau), \tau)| d \tau \\
& \leqslant C\left(1+\max _{(x, \tau) \in \Omega_{t}}|B(x, \tau)|\right) .
\end{aligned}
$$

It completes the proof.

Lemma 4. For each $t \in[0, T]$ the estimates

$$
\begin{aligned}
& \max _{0 \leqslant x \leqslant s(t)} \rho(x, t) \leqslant M \exp \left\{2 \max _{(x, \tau) \in \Omega_{t}}|B(x, \tau)|\right\}, \\
& \max _{0 \leqslant x \leqslant s(t)} \frac{1}{\rho(x, t)} \leqslant C\left[\exp \left\{2 \max _{(x, \tau) \in \Omega_{t}}|B(x, \tau)|\right\}+\exp \left\{4 \max _{(x, \tau) \in \Omega_{t}}|B(x, \tau)|\right\} \int_{0}^{t} \max _{0 \leqslant x \leqslant s(\tau)} \theta(x, \tau) d \tau\right]
\end{aligned}
$$

hold true.

Proof. We multiply identity $(12)$ by $\rho\left(\rho e^{B}\right)^{n-1}$, where $n$ is a natural number and integrate w.r.t. $x$ from 0 to $s(t)$ employing (1):

$$
\frac{1}{n} \frac{d}{d t} \int_{0}^{s(t)} \rho\left(\rho e^{B}\right)^{n} d x-\frac{1}{n} \frac{d s(t)}{d t} \rho_{2}(t)\left[\rho_{2}(t) e^{B(s(t), t)}\right]^{n}+\left.\frac{1}{n} \rho u\left(\rho e^{B}\right)^{n}\right|_{x=0} ^{x=s(t)}+\frac{R}{\mu} \int_{0}^{s(t)} \rho^{2}\left(\rho e^{B}\right)^{n} \theta d x=0 .
$$

By (5) and (7) we get

$$
\frac{1}{n} \frac{d}{d t} \int_{0}^{s(t)} \rho\left(\rho e^{B}\right)^{n} d x-\frac{1}{n} \frac{d s(t)}{d t} \rho_{2}(t)\left[\rho_{2}(t) e^{B(s(t), t)}\right]^{n}+\frac{R}{\mu} \int_{0}^{s(t)} \rho^{2} \theta\left(\rho e^{B}\right)^{n} d x=0 .
$$

The third term is non-negative and thus

$$
\frac{d}{d t} \int_{0}^{s(t)} \rho^{n+1} e^{n B} d x-\frac{d s(t)}{d t} \rho_{2}(t)\left[\rho_{2}(t) e^{B(s(t), t)}\right]^{n} \leqslant 0 .
$$

Integrating w.r.t. the time from 0 to $t$, we obtain

$$
\begin{aligned}
\int_{0}^{s(t)} \rho^{n+1} e^{n B} d x & \leqslant \int_{0}^{s_{0}} \rho_{0}^{n+1} e^{n B_{0}} d x+\int_{0}^{t} \frac{d s(\tau)}{d \tau} \rho_{2}^{n+1}(\tau) e^{n B(s(\tau), \tau)} d \tau \\
& \leqslant M^{n+1}\left(s_{0}+T M\right)\left[\max _{(x, \tau) \in \Omega_{t}} e^{B(x, \tau)}\right]^{n} .
\end{aligned}
$$


Then

$$
\int_{0}^{s(t)} \rho^{n+1} d x \leqslant C M^{n+1}\left[\max _{(x, \tau) \in \Omega_{t}} e^{B(x, \tau)}\right]^{n}\left[\min _{(x, \tau) \in \Omega_{t}} e^{B(x, \tau)}\right]^{-n} \leqslant C M^{n+1}\left[\exp \left\{2 \max _{(x, \tau) \in \Omega_{t}}|B(x, \tau)|\right\}\right]^{n} .
$$

Passing to the limit as $n \rightarrow \infty$ and employing the identity

$$
\lim _{n \rightarrow \infty}\left(\int_{a}^{b}|f(x)|^{n} d x\right)^{1 / n}=\max _{x \in[a, b]}|f(x)|
$$

we obtain estimate (18):

$$
\max _{0 \leqslant x \leqslant s(t)} \rho(x, t) \leqslant M \exp \left\{2 \max _{(x, \tau) \in \Omega_{t}}|B(x, \tau)|\right\} .
$$

In order to prove estimate $(19)$, we multiply identity $(13)$ by $\rho\left(\rho e^{B}\right)^{-n}$, where $n$ is a natural number, and integrate w.r.t. $x$ from 0 to $s(t)$ employing (1), (7):

$$
\begin{aligned}
\frac{1}{n+1} \frac{d}{d t} \int_{0}^{s(t)} \rho\left(\frac{1}{\rho e^{B}}\right)^{n+1} d x & -\frac{1}{n+1} \frac{d s(t)}{d t} \rho_{2}(t)\left(\frac{1}{\rho_{2}(t) e^{B(s(t), t)}}\right)^{n+1} \\
& +\left.\frac{1}{n+1} \rho u\left(\frac{1}{\rho e^{B}}\right)^{n+1}\right|_{x=0} ^{x=s(t)}=\frac{R}{\mu} \int_{0}^{s(t)} \rho e^{-B} \theta\left(\rho e^{B}\right)^{-n} d x .
\end{aligned}
$$

By (5) and (7) we get

$$
\frac{d}{d t} \int_{0}^{s(t)}\left(\frac{1}{\rho e^{B}}\right)^{n} \frac{1}{e^{B}} d x=\frac{d s(t)}{d t}\left(\frac{1}{\rho_{2}(t) e^{B(s(t), t)}}\right)^{n} \frac{1}{e^{B(s(t), t)}}+\frac{R(n+1)}{\mu} \int_{0}^{s(t)} e^{-2 B} \theta\left(\frac{1}{\rho e^{B}}\right)^{n-1} d x .
$$

Integrating w.r.t. the time from 0 to $t$, we arrive at

$$
\begin{aligned}
\int_{0}^{s(t)}\left(\frac{1}{\rho e^{B}}\right)^{n} \frac{1}{e^{B}} d x \leqslant & s_{0}\left(\frac{1}{m}\right)^{n}\left[\max _{(x, \tau) \in \Omega_{t}} e^{-B(x, \tau)}\right]^{n+1}+M T\left(\frac{1}{m}\right)^{n}\left[\max _{(x, \tau) \in \Omega_{t}} e^{-B(x, \tau)}\right]^{n+1} \\
& +\frac{R(n+1)}{\mu}\left[\max _{(x, \tau) \in \Omega_{t}} e^{-2 B(x, \tau)}\right] \int_{0}^{t} \int_{0}^{s(\tau)}\left(\frac{1}{\rho e^{B}}\right)^{n-1} \theta d x d \tau .
\end{aligned}
$$

Then

$$
\begin{aligned}
\int_{0}^{s(t)}\left(\frac{1}{\rho e^{B}}\right)^{n} d x \leqslant & \left(s_{0}+M T\right)\left(\frac{1}{m}\right)^{n}\left[\max _{(x, \tau) \in \Omega_{t}} e^{B(x, \tau)}\right]\left[\max _{(x, \tau) \in \Omega_{t}} e^{-B(x, \tau)}\right]^{n+1} \\
& +\frac{R(n+1)}{\mu}\left[\max _{(x, \tau) \in \Omega_{t}} e^{B(x, \tau)}\right]\left[\max _{(x, \tau) \in \Omega_{t}} e^{-2 B(x, \tau)}\right] \int_{0}^{t} \int_{0}^{s(\tau)}\left(\frac{1}{\rho e^{B}}\right)^{n-1} \theta d x d \tau \\
\leqslant & C\left(\frac{1}{m}\right)^{n} \exp \left\{(n+2) \max _{(x, \tau) \in \Omega_{t}}|B(x, \tau)|\right\}+\frac{R(n+1)}{\mu} \exp \left\{3 \max _{(x, \tau) \in \Omega_{t}}|B(x, \tau)|\right\} \\
& \cdot \int_{0}^{t} \max _{0 \leqslant x \leqslant s(\tau)} \theta(x, \tau) \int_{0}^{s(\tau)}\left(\frac{1}{\rho e^{B}}\right)^{n-1} d x d \tau .
\end{aligned}
$$

We denote

$$
y(t)=\left(\int_{0}^{s(t)}\left(\frac{1}{\rho e^{B}}\right)^{n} d x\right)^{1 / n} .
$$

Applying Hölder inequality to the last integral in 20 , employing the inequality $s(\tau) \leqslant s(T)$, we obtain

$$
\int_{0}^{s(t)}\left(\frac{1}{\rho e^{B}}\right)^{n-1} d x \leqslant\left(\int_{0}^{s(t)}\left(\frac{1}{\rho e^{B}}\right)^{(n-1) \cdot \frac{n}{n-1}} d x\right)^{\frac{n-1}{n}} \cdot\left(\int_{0}^{s(t)} 1^{n} d x\right)^{\frac{1}{n}} \leqslant y^{n-1}(t) \cdot \sqrt[n]{s(T)}
$$


Substituting the obtained estimate into $(20)$, we have

$$
\begin{aligned}
y^{n}(t) \leqslant & C\left(\frac{1}{m}\right)^{n} \exp \left\{(n+2) \max _{(x, \tau) \in \Omega_{t}}|B(x, \tau)|\right\} \\
& +\frac{R(n+1) \sqrt[n]{s(T)}}{\mu} \exp \left\{3 \max _{(x, \tau) \in \Omega_{t}}|B(x, \tau)|\right\} \int_{0}^{t} \max _{0 \leqslant x \leqslant s(\tau)} \theta(x, \tau) y^{n-1}(\tau) d \tau .
\end{aligned}
$$

In order to estimate function $y(t)$ we make use of the following lemma.

Lemma 5 ([22]). If a continuous non-negative on $[0, T]$ function $y(t)$ satisfies the inequality

$$
y^{n}(t) \leqslant a+b \int_{0}^{t} c(\tau) y^{n-1}(\tau) d \tau
$$

where $a, b=$ const $\geqslant 0, n=$ const $\geqslant 1, c(t)$ is a given non-negative function in class $L_{1}[0, T]$, then the estimate

holds true.

$$
y(t) \leqslant \sqrt[n]{a}+\frac{b}{n} \int_{0}^{t} c(\tau) d \tau
$$

If we apply estimate $(22)$ to inequality $(21)$, we obtain

$$
\begin{aligned}
y(t) \leqslant & \frac{\sqrt[n]{C}}{m} \exp \left\{\left(1+\frac{2}{n}\right) \max _{(x, \tau) \in \Omega_{t}}|B(x, \tau)|\right\} \\
& +\frac{R \sqrt[n]{s(T)}}{\mu}\left(1+\frac{1}{n}\right) \exp \left\{3 \max _{(x, \tau) \in \Omega_{t}}|B(x, \tau)|\right\} \int_{0}^{t} \max _{0 \leqslant x \leqslant s(\tau)} \theta(x, \tau) d \tau .
\end{aligned}
$$

Passing to the limit as $n \rightarrow \infty$, we get

$$
\max _{0 \leqslant x \leqslant s(t)} \frac{1}{\rho e^{B}} \leqslant \frac{1}{m} \exp \left\{\max _{(x, \tau) \in \Omega_{t}}|B(x, \tau)|\right\}+\frac{R}{\mu} \exp \left\{3 \max _{(x, \tau) \in \Omega_{t}}|B(x, \tau)|\right\} \int_{0}^{t} \max _{0 \leqslant x \leqslant s(\tau)} \theta(x, \tau) d \tau .
$$

It implies estimate 190 . The proof is complete.

Lemma 6 (Estimate of total energy). There exists a constant $C>0$ depending on boundary data and $T$ such that

$$
\max _{0 \leqslant t \leqslant T} \int_{0}^{s(t)}\left(\rho \theta+\frac{\rho u^{2}}{2}\right) d x \leqslant C .
$$

Proof. In order to estimate the total energy, we introduce the auxiliary function $A(x, t)$ as solution to the boundary value problem

$$
\begin{aligned}
& \rho\left(A_{t}+u A_{x}\right)=\kappa A_{x x},(x, t) \in \Omega_{T}, \\
& \left.A\right|_{x=0}=\theta_{1}(t),\left.\quad A\right|_{x=s(t)}=\theta_{2}(t),\left.\quad A\right|_{t=0}=\theta_{0}(x) .
\end{aligned}
$$

By the maximum principle we obtain

$$
0<m \leqslant A(x, t) \leqslant M<+\infty .
$$

The function

which takes values

$$
\varphi(x, t)=\theta(x, t) A^{-1}(x, t)
$$

satisfies by (3) and (23) the equation

$$
\left.\varphi\right|_{x=0}=\left.\varphi\right|_{x=s(t)}=1,\left.\varphi\right|_{t=0}=1,
$$

$$
A \rho\left(\varphi_{t}+u \varphi_{x}\right)=\kappa\left(A \varphi_{x}\right)_{x}+\kappa A_{x} \varphi_{x}+\mu u_{x}^{2}-R \rho A \varphi u_{x} .
$$

We multiply this equation by $\left(1-\frac{1}{\varphi}\right)$ and integrate w.r.t. $x$ from 0 to $s(t)$. Employing (23), we obtain

$$
\begin{gathered}
\frac{d}{d t} \int_{0}^{s(t)} A \rho(\varphi-\ln \varphi-1) d x+\int_{0}^{s(t)} \kappa A \frac{\varphi_{x}^{2}}{\varphi^{2}} d x+\int_{0}^{s(t)} \frac{1}{\varphi} \mu u_{x}^{2} d x \\
=\int_{0}^{s(t)}\left(\mu u_{x}^{2}-R \rho A \varphi u_{x}\right) d x+\int_{0}^{s(t)} \frac{1}{\varphi} R \rho A \varphi u_{x} d x
\end{gathered}
$$


Multiplying equation (2) by $u(x, t)$ and integrating w.r.t. $x$ from 0 to $s(t)$ employing (1), we find

$$
\frac{1}{2} \frac{d}{d t} \int_{0}^{s(t)} \rho u^{2} d x+\int_{0}^{s(t)} u_{x}\left(\mu u_{x}-p\right) d x=0 .
$$

We sum up the obtained identity with 25

$$
\frac{d}{d t} \int_{0}^{s(t)}\left(A \rho(\varphi-\ln \varphi-1)+\frac{\rho u^{2}}{2}\right) d x+\int_{0}^{s(t)} \kappa A \frac{\varphi_{x}^{2}}{\varphi^{2}} d x+\int_{0}^{s(t)} \mu \frac{u_{x}^{2}}{\varphi} d x=\int_{0}^{s(t)} R \rho A u_{x} d x .
$$

Let us estimate the terms in the right hand side:

$$
\int_{0}^{s(t)} R \rho A u_{x} d x \leqslant \int_{0}^{s(t)}\left(\frac{\mu u_{x}^{2}}{2 \varphi}+\frac{R^{2} A}{2 \mu} \rho^{2} A \varphi\right) d x \leqslant \frac{\mu}{2} \int_{0}^{s(t)} \frac{u_{x}^{2}}{\varphi} d x+\frac{R^{2} M}{2 \mu} \int_{0}^{s(t)} \rho^{2} \theta d x .
$$

As a result, we arrive at the inequality

$$
\frac{d}{d t} \int_{0}^{s(t)}\left(A \rho(\varphi-\ln \varphi-1)+\frac{\rho u^{2}}{2}\right) d x+\int_{0}^{s(t)} \kappa A \frac{\varphi_{x}^{2}}{\varphi^{2}} d x+\frac{\mu}{2} \int_{0}^{s(t)} \frac{u_{x}^{2}}{\varphi} d x \leqslant C \int_{0}^{s(t)} \rho^{2} \theta d x .
$$

Integrating this inequality w.r.t. the time, we get

$$
\begin{aligned}
\int_{0}^{s(t)}\left(A \rho(\varphi-\ln \varphi-1)+\frac{\rho u^{2}}{2}\right) d x & +\int_{0}^{t} \int_{0}^{s(\tau)} \kappa A \frac{\varphi_{x}^{2}}{\varphi^{2}} d x d \tau+\frac{\mu}{2} \int_{0}^{t} \int_{0}^{s(\tau)} \frac{u_{x}^{2}}{\varphi} d x d \tau \\
& \leqslant C\left(1+\int_{0}^{t} \int_{0}^{s(\tau)} \rho^{2} \theta d x d \tau\right) .
\end{aligned}
$$

In view of the inequality

$$
\varphi-\ln \varphi-1 \geqslant \frac{1}{2} \varphi-\ln 2
$$

and the boundedness of $A(x, t)$, by (24) and Lemma 1 we obtain

$$
\begin{aligned}
\frac{1}{2} \int_{0}^{s(t)} \rho \theta d x+\frac{1}{2} \int_{0}^{s(t)} \rho u^{2} d x+\int_{0}^{t} \int_{0}^{s(\tau)} \kappa A \frac{\varphi_{x}^{2}}{\varphi^{2}} d x d \tau+\frac{\mu}{2} \int_{0}^{t} \int_{0}^{s(\tau)} \frac{u_{x}^{2}}{\varphi} d x d \tau \leqslant \\
\leqslant C\left(1+\int_{0}^{t} \int_{0}^{s(\tau)} \rho^{2} \theta d x d \tau\right) .
\end{aligned}
$$

By estimate (17) in Lemma 3 we have

$$
\int_{0}^{t} \int_{0}^{s(\tau)} \rho^{2} \theta d x d \tau \leqslant C\left(1+\max _{(x, \tau) \in \Omega_{t}}|B(x, \tau)|\right) .
$$

Employing the inequality in Lemma 2, we obtain

$$
\int_{0}^{t} \int_{0}^{s(\tau)} \rho^{2} \theta d x d \tau \leqslant C\left(1+\max _{0 \leqslant \tau \leqslant t}\left(\int_{0}^{s(\tau)} \rho u^{2} d x\right)^{1 / 2}+\int_{0}^{t} \int_{0}^{s(\tau)} \rho \theta d x d \tau+\int_{0}^{t} \int_{0}^{s(\tau)} \rho u^{2} d x d \tau\right) .
$$

By applying this estimate and neglecting in the left hand side of (27) the integrals involving the derivatives, we get

$$
\begin{aligned}
\frac{1}{2} \int_{0}^{s(t)} \rho \theta d x & +\frac{1}{2} \int_{0}^{s(t)} \rho u^{2} d x \\
& \leqslant C\left(1+\max _{0 \leqslant \tau \leqslant t}\left(\int_{0}^{s(\tau)} \rho u^{2} d x\right)^{1 / 2}+\int_{0}^{t} \int_{0}^{s(\tau)} \rho \theta d x d \tau+\int_{0}^{t} \int_{0}^{s(\tau)} \rho u^{2} d x d \tau\right) .
\end{aligned}
$$

We introduce two functions on $[0, T]$ :

$$
a(t)=\max _{0 \leqslant \tau \leqslant t} \int_{0}^{s(\tau)} \rho(x, \tau) u^{2}(x, \tau) d x, \quad b(t)=\max _{0 \leqslant \tau \leqslant t} \int_{0}^{s(\tau)} \rho(x, \tau) \theta(x, \tau) d x .
$$


Then by (28) we get

$$
\frac{1}{2} a(t)+\frac{1}{2} b(t) \leqslant C\left(1+\sqrt{a(t)}+\int_{0}^{t} a(\tau) d \tau+\int_{0}^{t} b(\tau) d \tau\right) .
$$

Since $C \sqrt{a(t)} \leqslant C^{2}+\frac{a(t)}{4}$, it follows from 29 that

$$
a(t)+b(t) \leqslant C\left(1+\int_{0}^{t} a(\tau) d \tau+\int_{0}^{t} b(\tau) d \tau\right) .
$$

By Grönwall inequality it yields $a(t) \leqslant C, b(t) \leqslant C$ for each $t \in[0, T]$, where $C$ is a positive constant. Returning back to inequality (27), we obtain the following series of estimates

$$
\begin{aligned}
& \max _{0 \leqslant t \leqslant T} \int_{0}^{s(t)} \rho(x, t) u^{2}(x, t) d x \leqslant C, \\
& \max _{0 \leqslant t \leqslant T} \int_{0}^{s(t)} \rho(x, t) \theta(x, t) d x \leqslant C, \\
& \int_{0}^{T} \int_{0}^{s(t)}\left(\kappa A \frac{\varphi_{x}^{2}}{\varphi^{2}}+\frac{u_{x}^{2}}{\varphi}\right) d x d \tau \leqslant C .
\end{aligned}
$$

Estimates (30), 31) provide the estimate for the total energy.

\section{UPPER AND LOWER BOUNDS FOR DENSITY AND LOWER BOUNDS FOR TEMPERATURE}

Lemma 7. There exists a constant $M_{1}>0$ depending on boundary data and $T$ such that

$$
\max _{(x, t) \in \Omega_{T}} \rho(x, t) \leqslant M_{1} \text {. }
$$

Proof. Lemma 2 and estimate (30), (31) imply the boundedness of function $B(x, t)$. The inequality (18) in Lemma 4 completes the proof.

Lemma 8. There exists a constant $m_{2}>0$ depending on boundary data and $T$ such that

$$
\min _{(x, t) \in \Omega_{T}} \theta(x, t) \geqslant m_{2} \text {. }
$$

Proof. We write equation (3) for the temperature as follows

$$
\rho\left(\theta_{t}+u \theta_{x}\right)=\kappa \theta_{x x}+\mu\left(u_{x}-\frac{R \rho \theta}{2 \mu}\right)^{2}-\frac{R^{2} \rho^{2} \theta^{2}}{4 \mu} .
$$

Dividing by $\rho \theta^{2}$, in domain $\Omega_{T}$ we obtain the equation for the function $q(x, t)=1 / \theta(x, t)$ :

$$
q_{t}+u q_{x}-\frac{\kappa}{\rho} q_{x x}=\frac{R^{2} \rho}{4 \mu}-\left[\frac{2 \kappa}{\rho} \theta q_{x}^{2}+\frac{\mu}{\rho} q^{2}\left(u_{x}-\frac{R \rho \theta}{2 \mu}\right)^{2}\right] .
$$

We pass from function $q(x, t)$ to a new function $v(x, t)$, by the identity

$$
q(x, t)=v(x, t) e^{t} .
$$

Thanks to 35$)$, function $v(x, t)$ satisfies the equation

$$
v_{t}+u v_{x}-\frac{\kappa}{\rho} v_{x x}+v=\frac{R^{2} \rho}{4 \mu} e^{-t}-\left[\frac{2 \kappa}{\rho} \theta v_{x}^{2} e^{t}+\frac{\mu}{\rho} v^{2} e^{t}\left(u_{x}-\frac{R \rho \theta}{2 \mu}\right)^{2}\right] .
$$

By Lemma 7 and the non-negativeness of terms in the square brackets for function $v(x, t)$ we have the differential inequality

$$
v_{t}+u v_{x}-\frac{\kappa}{\rho} v_{x x}+v \leqslant C e^{-t}
$$

Suppose that function $v(x, t)$ attains its positive maximum at an interior point $\left(x_{0}, t_{0}\right)$ in domain $\Omega_{T}$ or as $t_{0}=T$. Then at this point

$$
v_{t} \geqslant 0, \quad v_{x}=0, \quad v_{x x} \leqslant 0 .
$$


By (36), (37) we obtain the estimate

$$
\max _{(x, t) \in \Omega_{T}} v(x, t) \leqslant C e^{-t_{0}} \leqslant C .
$$

Therefore, for each $(x, t) \in \Omega_{T}$ the inequalities

$$
\frac{q(x, t)}{e^{t}} \leqslant C, q(x, t) \leqslant C e^{t} \leqslant C e^{T}, \frac{1}{\theta(x, t)} \leqslant C e^{T}
$$

hold true or

The proof is complete.

$$
\theta(x, t) \geqslant m_{2}=\frac{1}{C e^{T}}
$$

Lemma 9. There exists a constant $m_{1}>0$ depending on boundary data and $T$ such that

$$
\min _{(x, t) \in \Omega_{T}} \rho(x, t) \geqslant m_{1}
$$

Proof. Estimates (24), (32) yield

$$
\int_{0}^{t} \int_{0}^{s(\tau)} \frac{\varphi_{x}^{2}}{\varphi^{2}} d x d \tau \leqslant C
$$

By Lemma 4, the estimate $|B(x, t)| \leqslant C$ and in view of 24 we have

$$
\begin{aligned}
\max _{0 \leqslant x \leqslant s(t)} \frac{1}{\rho(x, t)} & \leqslant C\left(1+\int_{0}^{t} \max _{0 \leqslant x \leqslant s(\tau)} \theta(x, \tau) d \tau\right) \\
& \leqslant C\left(1+\int_{0}^{t} \max _{0 \leqslant x \leqslant s(\tau)} A(x, \tau) \varphi(x, \tau) d \tau\right) \leqslant C+C M \int_{0}^{t} \max _{0 \leqslant x \leqslant s(\tau)} \varphi(x, \tau) d \tau .
\end{aligned}
$$

Taking into consideration the inequalities

$$
\begin{aligned}
& \varphi(x, t)=(\sqrt{\varphi(x, t)})^{2} \leqslant\left(1+\int_{0}^{s(t)}\left|(\sqrt{\varphi})_{x}\right| d x\right)^{2} \leqslant\left(1+\frac{1}{2} \int_{0}^{s(t)}\left|\frac{\varphi_{x}}{\sqrt{\varphi}}\right| d x\right)^{2} \\
& \leqslant\left[1+\frac{1}{2}\left(\int_{0}^{s(t)} \frac{\varphi_{x}^{2}}{\varphi^{2}} d x\right)^{1 / 2}\left(\int_{0}^{s(t)} \varphi d x\right)^{1 / 2}\right]^{2} \leqslant 2\left(1+\int_{0}^{s(t)} \frac{\varphi_{x}^{2}}{\varphi^{2}} d x \int_{0}^{s(t)} \varphi d x\right), \\
& \int_{0}^{s(t)} \varphi(x, t) d x=\int_{0}^{s(t)} \frac{\rho \theta}{\rho A} d x \leqslant \frac{1}{m} \max _{0 \leqslant x \leqslant s(t)} \frac{1}{\rho(x, t)} \int_{0}^{s(t)} \rho \theta d x \leqslant C \max _{0 \leqslant x \leqslant s(t)} \frac{1}{\rho(x, t)},
\end{aligned}
$$

by $(38)$ and $(39)$ we arrive at the inequality

$$
\max _{0 \leqslant x \leqslant s(t)} \frac{1}{\rho(x, t)} \leqslant C+C \int_{0}^{t} \max _{0 \leqslant x \leqslant s(\tau)} \frac{1}{\rho(x, \tau)} d \tau .
$$

Applying Grönwall inequality, we complete the proof.

In the above proof there were obtained the estimates

$$
\int_{0}^{t} \max _{0 \leqslant x \leqslant s(\tau)} \theta(x, \tau) d \tau \leqslant C+C \int_{0}^{t} \max _{0 \leqslant x \leqslant s(\tau)} \frac{1}{\rho(x, \tau)} d \tau, \quad \int_{0}^{s(t)} \varphi(x, t) d x \leqslant C \max _{0 \leqslant x \leqslant s(t)} \frac{1}{\rho(x, t)},
$$

which imply

$$
\begin{aligned}
& \int_{0}^{T} \max _{0 \leqslant x \leqslant s(t)} \theta(x, t) d t \leqslant C, \\
& \int_{0}^{s(t)} \theta(x, t) d x=\int_{0}^{s(t)} A(x, t) \varphi(x, t) d x \leqslant M \int_{0}^{s(t)} \varphi(x, t) d x \leqslant C, \\
& \int_{0}^{T} \int_{0}^{s(t)} \theta^{2}(x, t) d x d t \leqslant \int_{0}^{T} \max _{0 \leqslant x \leqslant s(t)} \theta(x, t) \int_{0}^{s(t)} \theta(x, t) d x d t \leqslant C .
\end{aligned}
$$




\section{ESTIMATES FOR DERIVATIVES}

Lemma 10. There exists a constant $C>0$ depending on boundary data and $T$ such that

$$
\max _{t \in[0, T]} \int_{0}^{s(t)} \rho(x, t) u^{2}(x, t) d x+\int_{0}^{T} \int_{0}^{s(t)} u_{x}^{2}(x, t) d x d t \leqslant C .
$$

Proof. We multiply equation (2) by $u(x, t)$ and integrate it w.r.t. $x$ from 0 to $s(t)$ employing (1), (5), and (7):

$$
\frac{1}{2} \frac{d}{d t} \int_{0}^{s(t)} \rho u^{2} d x+\mu \int_{0}^{s(t)} u_{x}^{2} d x-\int_{0}^{s(t)} R \rho \theta u_{x} d x=0 .
$$

Applying Young inequality with $\varepsilon$ and integrating w.r.t. $t$, we get

$$
\frac{1}{2} \int_{0}^{s(t)} \rho u^{2} d x+\mu \int_{0}^{t} \int_{0}^{s(\tau)} u_{x}^{2} d x d \tau \leqslant C_{1}+\frac{R^{2}}{\varepsilon} \int_{0}^{t} \int_{0}^{s(\tau)} \rho^{2} \theta^{2} d x d \tau+\varepsilon \int_{0}^{t} \int_{0}^{s(\tau)} u_{x}^{2} d x d \tau .
$$

Choosing $\varepsilon$ sufficiently small and applying Lemma 7 and inequality (42), we obtain

$$
\int_{0}^{s(t)} \rho u^{2} d x+\int_{0}^{t} \int_{0}^{s(\tau)} u_{x}^{2} d x d \tau \leqslant C .
$$

The proof is complete.

As a corollary of Lemmata 9 and 10 we obtain the estimate

$$
\max _{t \in[0, T]} \int_{0}^{s(t)} u^{2}(x, t) d x+\int_{0}^{T} \int_{0}^{s(t)} u_{x}^{2}(x, t) d x d t \leqslant C .
$$

Lemma 11. For each $t \in[0, T]$ the inequality

$$
\begin{aligned}
& \int_{0}^{s(t)} u_{x}^{2}(x, t) d x+\int_{0}^{t} \int_{0}^{s(\tau)}\left(u_{\tau}^{2}(x, \tau)+u_{x x}^{2}(x, \tau)\right) d x d \tau \\
& \leqslant C\left(1+\int_{0}^{t} \int_{0}^{s(\tau)} \theta_{x}^{2}(x, \tau) d x d \tau+\int_{0}^{t} \max _{x \in[0, s(\tau)]} \theta^{2}(x, \tau) \int_{0}^{s(\tau)} \rho_{x}^{2}(x, \tau) d x d \tau\right)
\end{aligned}
$$

holds true.

Proof. We represent equation (2) as

$$
\sqrt{\rho} u_{t}-\frac{1}{\sqrt{\rho}} \mu u_{x x}=-\sqrt{\rho} u u_{x}-R \sqrt{\rho} \theta_{x}-\frac{1}{\sqrt{\rho}} R \rho_{x} \theta .
$$

Hence,

$$
\int_{0}^{s(t)}\left(\rho u_{t}^{2}+\frac{1}{\rho} \mu^{2} u_{x x}^{2}-2 \mu u_{t} u_{x x}\right) d x=\int_{0}^{s(t)}\left(\sqrt{\rho} u u_{x}+R \sqrt{\rho} \theta_{x}+\frac{1}{\sqrt{\rho}} R \rho_{x} \theta\right)^{2} d x .
$$

Thus,

$$
\begin{aligned}
\int_{0}^{s(t)} \rho u_{t}^{2} d x & +\mu^{2} \int_{0}^{s(t)} \frac{1}{\rho} u_{x x}^{2} d x-2 \mu u_{t}(s(t), t) u_{x}(s(t), t)+\mu \frac{d}{d t} \int_{0}^{s(t)} u_{x}^{2} d x \\
& -\mu \frac{d s(t)}{d t} u_{x}^{2}(s(t), t) \leqslant 3 \int_{0}^{s(t)}\left(\rho u^{2} u_{x}^{2}+R^{2} \rho \theta_{x}^{2}+\frac{1}{\rho} R^{2} \rho_{x}^{2} \theta^{2}\right) d x .
\end{aligned}
$$

Since $u(s(t), t)=0$, then

$$
u_{x}(s(t), t) \frac{d s(t)}{d t}=-u_{t}(s(t), t)
$$

and by (46) it implies the inequality

$$
\begin{aligned}
\int_{0}^{s(t)} \rho u_{t}^{2} d x+\mu^{2} \int_{0}^{s(t)} \frac{1}{\rho} u_{x x}^{2} d x+\mu & \frac{d}{d t} \int_{0}^{s(t)} u_{x}^{2} d x+\mu \frac{d s(t)}{d t} u_{x}^{2}(s(t), t) \\
& \leqslant 3 \int_{0}^{s(t)}\left(\rho u^{2} u_{x}^{2}+R^{2} \rho \theta_{x}^{2}+\frac{1}{\rho} R^{2} \rho_{x}^{2} \theta^{2}\right) d x .
\end{aligned}
$$


We have

$$
u_{x}^{2}(x, t) \leqslant u_{x}^{2}\left(x_{0}, t\right)+\int_{x_{0}}^{x}\left|\left(u_{x}^{2}\right)_{x}\right| d x \leqslant u_{x}^{2}\left(x_{0}, t\right)+\int_{0}^{s(t)}\left|\left(u_{x}^{2}\right)_{x}\right| d x=u_{x}^{2}\left(x_{0}, t\right)+2 \int_{0}^{s(t)}\left|u_{x} u_{x x}\right| d x .
$$

Given any $\varepsilon>0$, we integrate this inequality w.r.t. $x_{0}$ from 0 to $s(t)$

$$
\begin{aligned}
u_{x}^{2}(x, t) & \leqslant \frac{1}{s(t)} \int_{0}^{s(t)} u_{x}^{2}(x, t) d x+2 \int_{0}^{s(t)}\left|u_{x} u_{x x}\right| d x \\
& \leqslant\left(\frac{1}{s_{0}}+\frac{1}{\varepsilon}\right) \int_{0}^{s(t)} u_{x}^{2} d x+\varepsilon \int_{0}^{s(t)} u_{x x}^{2} d x .
\end{aligned}
$$

Taking into consideration (48), the non-negativeness of the term $\mu \frac{d s(t)}{d t} u_{x}^{2}(s(t), t)$, the estimate

$$
\int_{0}^{s(t)} \rho u^{2} u_{x}^{2} d x \leqslant C \max _{0 \leqslant x \leqslant s(t)} u_{x}^{2}(x, t) \leqslant \frac{C}{\varepsilon} \int_{0}^{s(t)} u_{x}^{2} d x+C \varepsilon \int_{0}^{s(t)} u_{x x}^{2} d x
$$

Lemma 10 and the boundedness of $\rho(x, t)$ from above and below, by 47) we obtain 45 under appropriate choice of $\varepsilon>0$.

Lemma 12. For each $t \in[0, T]$ the inequality

$$
\int_{0}^{s(t)} \rho_{x}^{2}(x, t) d x \leqslant C+C \int_{0}^{t} \int_{0}^{s(\tau)} \frac{\theta_{x}^{2}}{\theta} d x d \tau+C \varepsilon \int_{0}^{t} \int_{0}^{s(\tau)} u_{x x}^{2} d x d \tau
$$

holds true.

Proof. By equation (1) we have

$$
(\ln \rho)_{t}+u(\ln \rho)_{x}+u_{x}=0 .
$$

Then equation (2) casts into the form

$$
(\rho u)_{t}+\left(\rho u^{2}\right)_{x}=-\mu\left[(\ln \rho)_{t}+u(\ln \rho)_{x}\right]_{x}-p_{x} .
$$

Hence,

$$
\rho\left[\left(u+\mu \frac{\rho_{x}}{\rho^{2}}\right)_{t}+u\left(u+\mu \frac{\rho_{x}}{\rho^{2}}\right)_{x}\right]+p_{x}=0 .
$$

We multiply this identity by $\left(u+\mu \rho_{x} / \rho^{2}\right)$ and integrate w.r.t. $x$ from 0 to $s(t)$ :

$$
\begin{aligned}
& \frac{1}{2} \frac{d}{d t} \int_{0}^{s(t)} \rho\left(u+\mu \frac{\rho_{x}}{\rho^{2}}\right)^{2} d x-\left.\frac{1}{2} \frac{d s(t)}{d t} \rho\left(u+\mu \frac{\rho_{x}}{\rho^{2}}\right)^{2}\right|_{x=s(t)}+ \\
& +\left.\frac{1}{2} \rho u\left(u+\mu \frac{\rho_{x}}{\rho^{2}}\right)^{2}\right|_{x=0} ^{x=s(t)}-\int_{0}^{s(t)} R \rho \theta u_{x} d x+\left.R \rho \theta u\right|_{x=0} ^{x=s(t)}+\int_{0}^{s(t)}(R \rho \theta)_{x} \mu \frac{\rho_{x}}{\rho^{2}} d x=0 .
\end{aligned}
$$

Since $u=0$ as $x=s(t)$ and $x=0$, the obtained identity becomes

$$
\begin{aligned}
\frac{1}{2} \frac{d}{d t} \int_{0}^{s(t)}\left(\rho u^{2}+2 \mu \frac{u \rho_{x}}{\rho}+\mu^{2} \frac{\rho_{x}^{2}}{\rho^{3}}\right) d x & -\int_{0}^{s(t)} R \rho \theta u_{x} d x+R \mu \int_{0}^{s(t)} \frac{\theta \rho_{x}^{2}}{\rho^{2}} d x \\
& +R \mu \int_{0}^{s(t)} \frac{\theta_{x} \rho_{x}}{\rho} d x-\left.\frac{\mu^{2}}{2} \frac{d s}{d t} \frac{\rho_{x}^{2}}{\rho^{3}}\right|_{x=s(t)}=0 .
\end{aligned}
$$

Employing Lemma 7, the replace the third term in the left hand side by a smaller one:

$$
\begin{gathered}
\frac{1}{2} \frac{d}{d t} \int_{0}^{s(t)}\left(\rho u^{2}+2 \mu \frac{u \rho_{x}}{\rho}+\mu^{2} \frac{\rho_{x}^{2}}{\rho^{3}}\right) d x-\int_{0}^{s(t)} R \rho \theta u_{x} d x+\frac{R \mu}{M_{1}^{2}} \int_{0}^{s(t)} \theta \rho_{x}^{2} d x \\
+R \mu \int_{0}^{s(t)} \frac{\theta_{x} \rho_{x}}{\rho} d x-\left.\frac{\mu^{2}}{2} \frac{d s}{d t} \frac{\rho_{x}^{2}}{\rho^{3}}\right|_{x=s(t)} \leqslant 0 .
\end{gathered}
$$

Let us estimate some of the terms. By equation (1) for $x=s(t)$, and (5), (7), we obtain

$$
\rho_{t}(s(t), t)=-\rho_{2}(t) u_{x}(s(t), t) .
$$


On the other hand, differentiating (7) w.r.t. $t$, we have

$$
\rho_{x}(s(t), t) \frac{d s(t)}{d t}+\rho_{t}(s(t), t)=\frac{d \rho_{2}(t)}{d t}, \quad \rho_{x}(s(t), t) \frac{d s(t)}{d t}=\frac{d \rho_{2}(t)}{d t}+\rho_{2}(t) u_{x}(s(t), t) .
$$

Then by condition (9), we get

$$
\begin{aligned}
\rho_{x}^{2}(s(t), t) \frac{d s(t)}{d t} & =\left(\frac{d \rho_{2}(t)}{d t}+\rho_{2}(t) u_{x}(s(t), t)\right)^{2} \frac{1}{d s(t) / d t} \\
& \leqslant 2\left(\frac{d \rho_{2}(t)}{d t}\right)^{2} \frac{1}{m}+\frac{2 M^{2}}{m} u_{x}^{2}(s(t), t) \leqslant C\left(1+u_{x}^{2}(s(t), t)\right) .
\end{aligned}
$$

By (48) it yields

$$
\rho_{x}^{2}(s(t), t) \frac{d s(t)}{d t} \leqslant C+\frac{C}{\varepsilon} \int_{0}^{s(t)} u_{x}^{2} d x+\varepsilon C \int_{0}^{s(t)} u_{x x}^{2} d x
$$

Thus,

$$
\frac{\mu^{2}}{2} \frac{d s(t)}{d t} \frac{\rho_{x}^{2}(s(t), t)}{\rho^{3}(s(t), t)} \leqslant C+\frac{C}{\varepsilon} \int_{0}^{s(t)} u_{x}^{2} d x+\varepsilon C \int_{0}^{s(t)} u_{x x}^{2} d x .
$$

Due to the boundedness of $\rho(x, t)$ from above and below, (40), 442), Lemma 10, and the estimates

$$
\begin{aligned}
& \int_{0}^{t} \int_{0}^{s(\tau)} R \rho \theta u_{x} d x d \tau \leqslant C \int_{0}^{t} \int_{0}^{s(\tau)} \theta^{2} d x d \tau+C \int_{0}^{t} \int_{0}^{s(\tau)} u_{x}^{2} d x d \tau \leqslant C, \\
& -R \mu \int_{0}^{s(t)} \frac{\theta_{x} \rho_{x}}{\rho} d x \leqslant \frac{C}{\varepsilon_{1}} \int_{0}^{s(t)} \frac{\theta_{x}^{2}}{\theta} d x+\varepsilon_{1} C \int_{0}^{s(t)} \theta \rho_{x}^{2} d x \\
& -\mu \int_{0}^{s(t)} \frac{u \rho_{x}}{\rho} d x \leqslant \frac{C}{\varepsilon_{2}} \int_{0}^{s(t)} u^{2} d x+\varepsilon_{2} C \int_{0}^{s(t)} \rho_{x}^{2} d x
\end{aligned}
$$

we integrate (49) w.r.t. the time and for an appropriate choice of $\varepsilon_{1}, \varepsilon_{2}$ we arrive at the statement of the lemma. The proof is complete.

\section{ESTIMATE FOR TEMPERATURE}

Lemma 13. There exists a constant $C>0$ such that for each $t \in[0, T]$ the estimate

$$
\max _{0 \leqslant \tau \leqslant t} \int_{0}^{s(\tau)} \theta^{2} d x+\int_{0}^{t} \int_{0}^{s(\tau)} \theta_{x}^{2} d x d \tau \leqslant C
$$

holds true.

Proof. We write the equation for the energy $\theta+\frac{1}{2} u^{2}$. In order to do it, we multiply equation (2) by $u$ and sum up with (3):

$$
\rho\left(\theta+\frac{u^{2}}{2}\right)_{t}+\rho u\left(\theta+\frac{u^{2}}{2}\right)_{x}=\mu\left(u_{x} u\right)_{x}+\kappa \theta_{x x}-(R \rho \theta u)_{x} .
$$

We multiply this identity by

$$
\theta+\frac{u^{2}}{2}-\frac{x \theta_{2}(t)}{s(t)}-\left(1-\frac{x}{s(t)}\right) \theta_{1}(t)
$$


and integrate w.r.t. $x$ from 0 to $s(t)$ :

$$
\begin{aligned}
\frac{1}{2} & \frac{d}{d t} \int_{0}^{s(t)} \rho\left(\theta+\frac{u^{2}}{2}\right)^{2} d x-\frac{1}{2} \frac{d s(t)}{d t} \rho_{2}(t) \theta_{2}^{2}(t) \\
& -\frac{d}{d t} \int_{0}^{s(t)} \rho\left(\theta+\frac{u^{2}}{2}\right)\left[\frac{x \theta_{2}(t)}{s(t)}+\left(1-\frac{x}{s(t)}\right) \theta_{1}(t)\right] d x+\frac{d s(t)}{d t} \rho_{2}(t) \theta_{2}^{2}(t) \\
& +\int_{0}^{s(t)} \rho\left(\theta+\frac{u^{2}}{2}\right)\left[\frac{x \theta_{2}(t)}{s(t)}+\left(1-\frac{x}{s(t)}\right) \theta_{1}(t)\right]_{t} d x+\frac{\theta_{2}(t)-\theta_{1}(t)}{s(t)} \int_{0}^{s(t)} \rho u\left(\theta+\frac{u^{2}}{2}\right) d x \\
& =-\mu \int_{0}^{s(t)} u u_{x} \theta_{x} d x-\mu \int_{0}^{s(t)} u^{2} u_{x}^{2} d x+\mu \frac{\theta_{2}(t)-\theta_{1}(t)}{s(t)} \int_{0}^{s(t)} u u_{x} d x-\kappa \int_{0}^{s(t)} \theta_{x}^{2} d x \\
& -\kappa \int_{0}^{s(t)} u u_{x} \theta_{x} d x+\kappa \frac{\theta_{2}(t)-\theta_{1}(t)}{s(t)}\left(\theta_{2}(t)-\theta_{1}(t)\right)+R \int_{0}^{s(t)} \rho \theta u \theta_{x} d x+R \int_{0}^{s(t)} \rho \theta u^{2} u_{x} d x \\
& -R \frac{\theta_{2}(t)-\theta_{1}(t)}{s(t)} \int_{0}^{s(t)} \rho \theta u d x .
\end{aligned}
$$

Let us estimate some terms in (51):

$$
\begin{aligned}
& \frac{\theta_{2}(t)-\theta_{1}(t)}{s(t)} \int_{0}^{s(t)} \rho u\left(\theta+\frac{u^{2}}{2}\right) d x \leqslant C \int_{0}^{s(t)} u^{2} d x+C \int_{0}^{s(t)} \rho\left(\theta+\frac{u^{2}}{2}\right)^{2} d x, \\
& \int_{0}^{s(t)} u u_{x} \theta_{x} d x \leqslant \frac{1}{\varepsilon} \int_{0}^{s(t)} u^{2} u_{x}^{2} d x+\varepsilon \int_{0}^{s(t)} \theta_{x}^{2} d x, \\
& R \int_{0}^{s(t)} \rho \theta u \theta_{x} d x \leqslant \frac{R}{\varepsilon} \int_{0}^{s(t)} \rho^{2} \theta^{2} u^{2} d x+\varepsilon R \int_{0}^{s(t)} \theta_{x}^{2} d x, \\
& R \int_{0}^{s(t)} \rho \theta u^{2} u_{x} d x \leqslant R \int_{0}^{s(t)} \rho^{2} \theta^{2} u^{2} d x+R \int_{0}^{s(t)} u^{2} u_{x}^{2} d x .
\end{aligned}
$$

Other terms can be estimated in obvious way. Integrating (51) w.r.t. the time, we obtain the inequality

$$
\begin{aligned}
& \frac{1}{4} \int_{0}^{s(t)} \rho\left(\theta+\frac{u^{2}}{2}\right)^{2} d x+\frac{\kappa}{2} \int_{0}^{t} \int_{0}^{s(\tau)} \theta_{x}^{2} d x d \tau \\
& \quad \leqslant C+M_{3} \int_{0}^{t} \int_{0}^{s(\tau)} u^{2} u_{x}^{2} d x d \tau+C \int_{0}^{t} \int_{0}^{s(\tau)} \rho^{2} \theta^{2} u^{2} d x d \tau+C \int_{0}^{t} \int_{0}^{s(\tau)} \rho\left(\theta+\frac{u^{2}}{2}\right)^{2} d x d \tau,
\end{aligned}
$$

where $C, M_{3}$ are positive constants depending on $T$, initial and boundary data.

We multiply (2) by $4 u^{3}$, integrate w.r.t. $x$ from 0 to $s(t)$ and employ (1)

$$
\frac{d}{d t} \int_{0}^{s(t)} \rho u^{4} d x+12 \mu \int_{0}^{s(t)} u^{2} u_{x}^{2} d x=12 R \int_{0}^{s(t)} \rho \theta u^{2} u_{x} d x
$$

We apply Cauchy inequality to the right hand side

$$
\frac{d}{d t} \int_{0}^{s(t)} \rho u^{4} d x+12 \mu \int_{0}^{s(t)} u^{2} u_{x}^{2} d x \leqslant 6 \mu \int_{0}^{s(t)} u^{2} u_{x}^{2} d x+\frac{6 R^{2}}{\mu} \int_{0}^{s(t)} \rho^{2} \theta^{2} u^{2} d x .
$$

We integrate $(53)$ w.r.t. the time to obtain

$$
\int_{0}^{s(t)} \rho u^{4} d x+6 \mu \int_{0}^{t} \int_{0}^{s(\tau)} u^{2} u_{x}^{2} d x d \tau \leqslant C+C \int_{0}^{t} \int_{0}^{s(\tau)} \rho^{2} \theta^{2} u^{2} d x d \tau .
$$

We multiply (54) by $M_{3} / \mu$ and sum up with (52)

$$
\begin{aligned}
\frac{1}{4} \int_{0}^{s(t)} \rho\left(\theta+\frac{u^{2}}{2}\right)^{2} d x & +\frac{\kappa}{2} \int_{0}^{t} \int_{0}^{s(\tau)} \theta_{x}^{2} d x d \tau+\frac{M_{3}}{\mu} \int_{0}^{s(t)} \rho u^{4} d x+5 M_{3} \int_{0}^{t} \int_{0}^{s(\tau)} u^{2} u_{x}^{2} d x d \tau \\
& \leqslant C+C \int_{0}^{t} \int_{0}^{s(\tau)} \rho^{2} \theta^{2} u^{2} d x d \tau+C \int_{0}^{t} \int_{0}^{s(\tau)} \rho\left(\theta+\frac{u^{2}}{2}\right)^{2} d x d \tau
\end{aligned}
$$


In what follows we shall make use of the estimates

$$
\theta^{2}(x, t)=\theta_{1}^{2}(t)+\int_{0}^{x}\left(\theta^{2}\right)_{x} d x \leqslant \theta_{1}^{2}(t)+2 \int_{0}^{s(t)}\left|\theta \theta_{x}\right| d x \leqslant \theta_{1}^{2}(t)+\frac{1}{\varepsilon} \int_{0}^{s(t)} \theta^{2} d x+\varepsilon \int_{0}^{s(t)} \theta_{x}^{2} d x .
$$

Integrating w.r.t. the time and employing $(42)$, we get

$$
\int_{0}^{t} \max _{0 \leqslant x \leqslant s(\tau)} \theta^{2}(x, \tau) d \tau \leqslant C(\varepsilon)+\varepsilon \int_{0}^{t} \int_{0}^{s(\tau)} \theta_{x}^{2} d x d \tau .
$$

Then employing (44) and (56) we estimate as follows

$$
\begin{aligned}
\int_{0}^{t} \int_{0}^{s(\tau)} \rho^{2} \theta^{2} u^{2} d x d \tau & \leqslant M_{1}^{2} \int_{0}^{t}\left[\max _{0 \leqslant x \leqslant s(\tau)} \theta^{2}(x, \tau) \int_{0}^{s(\tau)} u^{2}(x, \tau) d x\right] d \tau \\
& \leqslant M_{1}^{2} \max _{0 \leqslant \tau \leqslant t} \int_{0}^{s(\tau)} u^{2}(x, \tau) d x \cdot \int_{0}^{t} \max _{0 \leqslant x \leqslant s(\tau)} \theta^{2}(x, \tau) d \tau \leqslant C+C \varepsilon \int_{0}^{t} \int_{0}^{s(\tau)} \theta_{x}^{2} d x d \tau .
\end{aligned}
$$

Employing this estimate for an appropriate choice of $\varepsilon$ and applying Grönwall inequality, by (55) we complete the proof.

Lemma 14. There exist constants $C_{1}, C_{2}, C_{3}, M_{2}>0$ depending on $T$, initial and boundary data such that for each $t \in[0, T]$ the estimates

$$
\begin{aligned}
& \int_{0}^{s(t)} u_{x}^{2}(x, t) d x+\int_{0}^{t} \int_{0}^{s(\tau)} u_{\tau}^{2} d x d \tau+\int_{0}^{t} \int_{0}^{s(\tau)} u_{x x}^{2} d x d \tau \leqslant C_{1}, \\
& \int_{0}^{s(t)} \rho_{x}^{2}(x, t) d x+\int_{0}^{s(t)} \rho_{t}^{2}(x, t) d x \leqslant C_{2}, \\
& \int_{0}^{s(t)} \theta_{x}^{2}(x, t) d x+\int_{0}^{t} \int_{0}^{s(\tau)} \theta_{\tau}^{2} d x d \tau+\int_{0}^{t} \int_{0}^{s(\tau)} \theta_{x x}^{2} d x d \tau \leqslant C_{3}, \\
& \max _{(x, t) \in \Omega_{T}} \theta(x, t) \leqslant M_{2} .
\end{aligned}
$$

hold true.

Proof. Thanks to the boundedness of $\theta(x, t)$ from below, by employing Lemma 13 we have

$$
\int_{0}^{t} \int_{0}^{s(\tau)} \frac{\theta_{x}^{2}}{\theta} d x d \tau \leqslant C
$$

Substituting this estimate into the statement of Lemma 12, we obtain

$$
\int_{0}^{s(t)} \rho_{x}^{2}(x, t) d x \leqslant C+C \varepsilon \int_{0}^{t} \int_{0}^{s(\tau)} u_{x x}^{2} d x d \tau
$$

Now estimate (57) follows from Lemmata 11, 13, (56), (60).

By (60) and (57) for each $t \in[0, T]$ we have

$$
\int_{0}^{s(t)} \rho_{x}^{2}(x, t) d x \leqslant C
$$

Equation (1) yields that for each $t \in[0, T]$

$$
\int_{0}^{s(t)} \rho_{t}^{2}(x, t) d x \leqslant C .
$$

Estimates (58), (59) can be obtained from equation (3) in the standard way by employing obtained estimates for $\rho$ and $u$.

Once we have apriori estimates proven in Lemmata 1-14, the estimates in the theorem in Hölder norms can be obtained by the approach presented in [25]. 


\section{BIBLIOGRAPHY}

1. J. Serrin. On the uniqueness of compressible fluid motion // Arch. Rat. Mech. Anal. 3:3, 271-288 (1959).

2. D. Graffi. Il teorema di unicita nella dinamica dei fluidi compressibli // J. Ration. Mech. Anal. 2, 99-106 (1953).

3. J. Nash. Le problème de Cauchy pour les équations différentielles d'un fluide général // Bull. Soc. Math. France. 90, 487-497 (1962).

4. N. Itaya. The existence and unicueness of the solution of the equations describing compressible viscous fluid flow // Proc. Japan Acad. 46:4, 379-382 (1970).

5. A.I. Vol'pert, S.I. Khudyaev. On the Cauchy problem for composite systems of nonlinear differential equations // Matem. Sbornik. 87:4, 504-528 (1972). [Math. USSR-Sbornik, 16:4, 517-544 (1972).]

6. V.A. Solonnikov. Solvability of the initial-boundary-value problem for the equations of motion of a viscous compressible fluid // Zap. Nauchn. Sem. LOMI. 56, 128-142 (1976). [J. Soviet Math. 14:2, 1120-1133 (1980).]

7. A. Tani. On the first initial-boundary value problem of compressible viscous fluid motion // Publ. Res. Inst. Math. Sci. Kyoto Univ. 3:1, 193-253 (1977).

8. Ja.I. Kanel'. On a model system of equations of one-dimensional gas motion // Differ. Uravn. 4:4, 721-734 (1968). (in Russian).

9. N. Itaya. On the temporally global problem of the generalized Burgers equation // J. Math. Kyoto Univ. 14:1, 129-177 (1974).

10. N. Itaya. A servey on the generalized Burgers equation with a pressure model term // J. Math. Kyoto Univ. 16:1, 223-240 (1976).

11. A. Tani. On the first initial-boundary value problem of the generalized Burgers equation // Publ. Res. Inst. Math. Sci. Kyoto Univ. 10:1, 209-233 (1974).

12. A.V. Kazhikhov. On global solvability of one-dimensional boundary value problems for equations of viscous heat-conducting gas // Continuous medium dynamics. Collection of scientific works. AS USSR, Siber. Branch. 24, 45-61 (1976). (in Russian).

13. A.V. Kazhikhov. Some issues in theory of Navier-Stokes equations of compressible liquid // Continuous medium dynamics. Collection of scientific works. AS USSR, Siber. Branch. 38, 33-47 (1979). (in Russian).

14. A.V. Kazhikhov. On theory of boundary value problems for equations of one-dimensional nonstationary motion of viscous heat-conducting gas // Continuous medium dynamics. Collection of scientific works. AS USSR, Siber. Branch. 50, 37-62 (1981). (in Russian).

15. A.V. Kazhikhov. Cauchy problem for viscous gas equations // Sibir. Matem. Zhurn. 23:1, 60-64 (1982). [Sib. Math. J. 23, 44-49 (1982).]

16. A.V. Kazhikhov, V.V. Shelukhin. Unique global solution with respect to time of initial-boundary value problems for one-dimensional equations of a viscous gas // Prikl. Matem. Mekh. 41:2, 282291 (1977). [J. Appl. Math. Mech. V41:2, 273-282 (1977).]

17. V.V. Shelukhin. Periodic flows of viscous gas // Continuous medium dynamics. Collection of scientific works. AS USSR, Siber. Branch. 42, 80-102 (1979). (in Russian).

18. V.V. Shelukhin. Existence of periodic solutions of the generalized Burgers system // Prikl. Matem. Mekh. 43:6, 992-997 (1979). [J. Appl. Math. Mech. 43:6, 1073-1079 (1981).]

19. V.V. Shelukhin. Bounded almost periodic solutions to viscous gas equations // Continuous medium dynamics. Collection of scientific works. AS USSR, Siber. Branch. 44, 147-162 (1980). (in Russian).

20. S.Ya. Belov. Global solvability of percolation problem for Burgers equations of compressible liquid // Continuous medium dynamics. Collection of scientific works. AS USSR, Siber. Branch. 50, 3-14 (1981). (in Russian).

21. V.A. Vaigant. Inhomogeneous boundary value problems for equations of viscous heat-conducting gas // Continuous medium dynamics. Collection of scientific works. AS USSR, Siber. Branch. 97, 3-21 (1990). (in Russian).

22. V.A. Vaigant. Existence problem for global solution to Navier-Stokes equations of compressible continuous media. Dissert. Doct. Phys.-Math. Sci. Barnaul, Altai State Univ. (1998). (in Russian). 
23. A.V. Kazhikhov, I.A. Kaliev. Well-definiteness of a model of phase transition gas-solid state // Preprint. Novgorod State University, Inst. Diskr. Math. Inform. 43, 32 pp. (1999). (in Russian).

24. I.A. Kaliev, A.V. Kazhikhov. Well-posedness of a gas-solid phase transition problem // J. Math. Fluid Mech. 1:3, 282-308 (1999).

25. S.N. Antontsev, A.V. Kazhikhov, V.N. Monakhov. Boundary value problems in mechanics of nonhomogeneous fluids. Nauka, Novosibirsk (1983). [Stud. Math. Appl. 22. North-Holland, Amsterdam (1990).]

26. I.A. Kaliev, M.S. Podkuiko. On a boundary value problem for the equations of a viscous heatconducting gas in noncylindrical domains shrinking in time // Differ. Uravn. 42:10, 1356-1374 (2006). [Differ. Equ. 42:10, 1426-1446 (2006).]

27. I.A. Kaliev, M.S. Podkuiko. Nonhomogeneous boundary value problems for equations of viscous heat-conducting gas in time-decreazing non-rectangular domains // J. Math. Fluid Mech. 10:2, 176-202 (2008).

Ibragim Adietovich Kaliev,

Sterlitamak Brach of Bashkir State University,

Lenin av., 47a,

453103, Sterlitamak, Russia

E-mail: kalievia@mail.ru

Andrey Alexandrovich Shukhardin,

Sterlitamak Brach of Bashkir State University,

Lenin av., 47a,

453103, Sterlitamak, Russia

E-mail: shukhardinaa@gmail.com

Gul'nara Sagyndykovna Sabitova,

Sterlitamak Brach of Bashkir State University,

Lenin av., 47a,

453103, Sterlitamak, Russia

E-mail: sabitovags@mail.ru 